\title{
Discussion of paper by S. Aime, N. D. Eisenmenger and T. A. P. Engels, entitled 'A model for failure in thermoplastic elastomers based on Eyring kinetics and network connectivity'
}

Aime, S.; Eisenmenger, N. D.; Engels, T. A. P.; Hassager, Ole; Watanabe, Hiroshi; Creton, Costantino; Colby, Ralph; Rubinstein, Michael; van Ruymbeke, Evelyne

Published in:

Journal of Rheology

Link to article, DOI:

$10.1122 / 1.5008883$

Publication date:

2017

Document Version

Peer reviewed version

Link back to DTU Orbit

Citation (APA):

Aime, S., Eisenmenger, N. D., Engels, T. A. P., Hassager, O., Watanabe, H., Creton, C., Colby, R., Rubinstein, M., \& van Ruymbeke, E. (2017). Discussion of paper by S. Aime, N. D. Eisenmenger and T. A. P. Engels, entitled 'A model for failure in thermoplastic elastomers based on Eyring kinetics and network connectivity'. Journal of Rheology, 61(6), 1343-1344. https://doi.org/10.1122/1.5008883

\section{General rights}

Copyright and moral rights for the publications made accessible in the public portal are retained by the authors and/or other copyright owners and it is a condition of accessing publications that users recognise and abide by the legal requirements associated with these rights.

- Users may download and print one copy of any publication from the public portal for the purpose of private study or research.

- You may not further distribute the material or use it for any profit-making activity or commercial gain

- You may freely distribute the URL identifying the publication in the public portal 
Discussion of paper by S. Aime, N. D. Eisenmenger and T. A. P. Engels, entitled 'A model for failure in thermoplastic elastomers based on Eyring kinetics and network connectivity'

By:Aime, S (Aime, S.); Eisenmenger, ND (Eisenmenger, N. D.); Engels, TAP (Engels, T. A. P.); Hassager, O (Hassager, Ole); Watanabe, H (Watanabe, Hiroshi); Creton, C (Creton, Costantino); Colby, R (Colby, Ralph); Rubinstein, M (Rubinstein, Michael); van Ruymbeke, E (van Ruymbeke, Evelyne)

\section{Questions JOR - Paper presented by S. Aime}

\section{Ole Hassager:}

How homogeneous is the sample failure?

Answer: At low T, you see stress whitening, possibly due to crazing (the tensile bars are rather transparent before the tensile test). This is not the case at high $\mathrm{T}$, where the sample looks transparent even after failure.

It sounds like 2 mechanisms are at play?

Answer: In our experiments we clearly see differences in the macroscopic sample morphology leading up to failure, which might contribute to the mismatch between experimental results and model predictions observed at room $\mathrm{T}$ (as mentioned in the paper). Nevertheless, we do believe that the failure in the end is dominated by a single mechanism: temperature and stress activated rupture of the connecting domains.

Is the stress an engineering or a true stress?

Answer: It is a true stress (we assume a homogeneous stress). For more details, see Sec. IV B in the paper.

\section{Hiroshi Watanabe:}

What about your associating/dissociating process? As mathematically described in your presentation, it seems that since you allow the bonds to break spontaneously, you will obtain bond breaking even if there is no stress applied?

Answer: Note that the bond-breakage dependence on stress is described by a sine hyperbolic (cf. Eq. 1 in the paper) which we simplified to a simple exponential dependence in case of large stresses. The original sine hyperbolic has an asymptote at zero stress (cf. Fig. 9).

\section{Costantino Creton:}

If you allow bonds to break, you can get breaking at zero stress, strain? You should do creep experiments to better understand the rupture mechanisms in the low stress/long time regime. If your lifetime prediction is correct (fracture for a constant damage) it should be creep dominated and you should be able to use the parameters obtained from creep experiments to predict the failure time in uniaxial extension as it was done in the group of Leon Govaert for glassy polymers. There are changes of structure, due to time but also due to the applied strain. This last origin is not taken into account in your model. 
Answer: Irrespective of the changes that can occur in the morphology during loading, and therefore of the exact morphology, we did find a strong temperature and stress dependence of the failure time in all cases. Being well familiar with the work of Govaert et al. we did perform some explorative creep experiments and find a qualitatively consistent behavior and we belief that indeed failure in both tests is governed by the same failure mechanism (cf. Sec. V B in the paper). To quantitatively connect the failure under a constant applied strain rate and constant applied stress a larger set of experiments is required. Performing such a full characterization was outside the scope of the current work.

\section{Daniel Read:}

Is there a speed-up of the breaking of remaining bonds, when a bond breaks, e.g. because of increased force in the other bonds at fixed stress?

Answer: Yes, and we show this in our simulations. In the initial section (Sec. IV) of the manuscript we use a simplified analytical approach that uses a scalar value for the overall connectivity as a critical threshold for failure that cannot take such details into account. In the second part (Sec. V), however, a numeral model is employed and local connectivity is explicitly taken into account (cf. Fig. 7). We show that especially for the lower molecular weights (poorly connected), stresses can be quite heterogeneous and breakage of bonds will result in local stress amplification (cf. Fig. 11).

\section{Ralph Colby:}

Why do you care about the chain length? Since your model is a kinetic model, why don't you simply change the activation energy?

Please note that we indeed propose that the kinetics of bond breakage are a key ingredient to understand failure (cf. Fig. 12). We however also show that connectivity is an important factor as well (cf. Fig. 3). Higher molecular weights allow for more bonds to be broken before failure is observed (cf. Fig. 11), hence increasing molecular weight is for example a very straightforward way to improve the lifetime of these systems.

\section{Michael Rubinstein:}

Have you tried to orient the sample? What is happening if you stretch a bit the sample, then wait, then stretch it, eventually in another direction?

We didn't try. We do expect that sample morphology (and anisotropic morphology for that matter) will play a role in the eventual failure, but we also believe that irrespective of the exact morphology the failure will be governed by the ingredients we identified in our paper: kinetics and connectivity. The proposed testing protocol where loads are applied in a non-continuous manner and even non-unidirectional fashion will be required if one wants to develop a model that allows for the quantitative prediction of failure for any arbitrary loading case, but this is well beyond the scope of our current paper.

\section{Evelyne van Ruymbeke:}


One of your experimental observations is that the elongation at break decreases with decreasing strain rate. How general is this observation?

You explain very nicely the effect of stress concentration and the breaking of weakest region. I wonder if with such systems, new bonds could also appear during the tensile test, which could "delocalize" this bond breaking? Could you take into account in your model the creation of these new bonds?

Answer: We believe that this decrease in elongation at failure for decreasing strain rates is a generic feature of thermoplastic elastomers that have a larger amount of soft-blocks. We have observed this behavior for urethane, ester and amide based block-copolymers (data not shown in the manuscript). Whether bonds can reform during deformation is something we cannot answer in detail, but we think is relatively unlikely for the systems we are discussing in this paper. Our model in its current form cannot take this into account. 